\title{
Assessment of clinicopathological features, evaluation of treatment, and prognosis of clear cell and serous papillary endometrial carcinoma
}

\author{
Mehmet Kulhan ${ }^{1,2}$, Gozde Kulhan 1, 2, Umit Nayki ${ }^{2}$, Cenk Nayki², Pasa Ulug ${ }^{2}$, \\ Mehmet Sipahi ${ }^{1}$, Yusuf Yildirim ${ }^{1,2}$ \\ ${ }^{1}$ Tepecik Obstetrics and Gynaecology Training and Research Hospital, Obstetrics and Gynaecology Unit, Izmir, Turkey \\ ${ }^{2}$ Erzincan University Medical Faculty, Gynaecology and Obstetrics Department, Erzincan, Turkey
}

\begin{abstract}
Objectives: We aimed to evaluate and compare the clinical and pathological features, diagnosis, treatment, and prognosis of uterine papillary serous carcinoma (UPSC) and clear-cell carcinoma (CC).

Material and methods: Thirty-four patients who were operated on for UPSC and CC carcinoma at the İzmir Tepecik Research and Teaching Hospital, between January 1983 and December 2014, were included. Patients were evaluated for the following factors: age, gravidity, parity, preoperative CA-125, tumor size, myometrial and lymphovascular invasion, lymph node tumor metastasis, presence of atypical cells in peritoneal cytology, pathology results, operation types, whether or not they received postoperative adjuvant therapy, prognosis, and death rate.

Results: The stage of the disease was the main factor affecting disease-free and the overall survival. Cisplatin-based chemotherapy was partially effective in patients with postoperative recurrence, while postoperative adjuvant radiotherapy proved to be more effective in preventing relapse.

Conclusion: The studied parameters generally were concordant with the literature but, due to the relatively small sample size, more comprehensive and multicenter studies are needed to generate valid results.
\end{abstract}

Key words: endometrial tumors, clear-cell carcinoma, papillary cell carcinoma

Ginekologia Polska 2016; 87, 8: 570-574

\section{INTRODUCTION}

Endometrial cancer is the most common malignancy of the female genital tract. Understanding the management strategies for this cancer is important to identify high-risk patients and reduce the risks associated with cancer and early diagnosis. Uterine serous papillary carcinoma (UPSC) and clear-cell carcinoma (CC), which tend to occur in older patients, are rare but very aggressive type II endometrial cancers. In 1983, Bokhman developed a hypothesis that there are two histological types of endometrial carcinoma and they have significantly different risk factors [1]. Type 1 or endometrioid carcinomas are estrogen-dependent tumors and they occur on the basis of hyperplasia. Type 2 or non-endometrioid tumors are usually seen in older patients and are less differentiated and more aggressive.
Type 2 tumors are independent of estrogen stimulation and do not have precursor lesions [2]. Type 2 cancers affect only $10-20 \%$ of patients with endometrial cancer but are responsible for $50 \%$ of deaths from endometrial cancer. The most common subgroups of type 2 endometrial cancer are uterine papillary serous carcinoma and clear-cell carcinoma [3]. UPSC is the most common type 2 histologically and accounts for $17-22 \%$ of all endometrial cancer cases [3,4]. Endometrial CC is rare and constitutes $1-6 \%$ of all endometrial cancer cases $[3,4]$. Due to the increasing incidence of endometrial carcinomas, it would require serious studies to determine risk factors, screening methods for early diagnosis, the most effective treatment methods, as well as survival and disease prognosis. The aim of the study was to evaluate the clinical and pathological 
features, treatment, and prognosis of serous papillary and clear-cell uterine cancers.

\section{MATERIAL AND METHODS}

Thirty-four patients who were operated on due to serous papillary or clear cell types of endometrial carcinoma at the İzmir Tepecik Research and Teaching Hospital, between January 1983 and December 2014, were included in the study. Clinical and pathological and surgical data were collected. All patients were evaluated for survival and disease-free survival. The following clinical data were collected from patient medical, surgical, pathological, and chemotherapy reports: demographic characteristics, presenting symptoms, serum CA-125 level, date and type of the surgical procedure, presence or absence of residual tumor after surgery, number of excised and positive lymph nodes, presence or absence of ascites, tumor pathological characteristics (grade and size), type of first-line chemotherapy, date of recurrence, treatment after recurrence, date of the last medical examination, and date of death. The revised 2009 FIGO (Federation of Gynecology and Obstetrics) staging system was used. The patients were classified as 'not staged' if only a total hysterectomy with unilateral or bilateral salpingo-oophorectomy with or without omentectomy was performed. Complete staging was defined as pelvic washing, peritoneal biopsy, omentectomy, bilateral pelvic and para-aortic lymph node dissection with bilateral salpingo-oophorectomy, and total abdominal hysterectomy. Optimal debulking was defined as a procedure which left a maximum residual tumor of $<1 \mathrm{~cm}$ in diameter. The patients returned for a follow-up every three months for the first 2 years, every 6 months for the next 3 years, and annually thereafter. Computed tomography or magnetic resonance imaging was performed annually. The survival analysis was based on the Kaplan-Meier method, and the results were compared using the log-rank test. Progression free survival (PFS) was defined as the time from the date of the primary surgery to detection of recurrence or the latest observation. OS was defined as the time from the date of primary surgery to death or the latest observation. The chi-square test and Student's $t$ test for unpaired data were used for statistical analyses. Cox regression analysis was used to determine factors affecting the survival, and the results are presented as hazard ratios (HRs), with $95 \%$ confidence intervals (Cls). All statistical analyses were performed using Statistical Package for the Social Sciences (SPSS ver. 11.5). The $p$-value of $<0.05$ was considered as statistically significant.

\section{RESULTS}

Mean patient age was 59.9 years at diagnosis. Age distribution was between 40 and 74 years. Postmenopau- sal bleeding was the most common complaint in both groups. One infertile patient was identified in each group. Mean parity distribution was between $0-6(3.27 \pm 1.61)$ and 0-8 (3.33 \pm 1.95$)$ in patients with CC and UPSC, respectively. As far as internal diseases and other cancers were concerned, diabetes mellitus was detected in 10 (43.41\%) and 3 (27.3\%) patients from the UPSC and CC groups, respectively. Hypertension was detected in 9 (39.04\%) patients from the UPSC group and in 1 (9.1\%) patient from the CC group. A history of breast cancer was identified in 2 subjects from the UPSC group. Demographic characteristics of the patients are summarized in Table 1.

Table 1. Demographic and morphological features

\begin{tabular}{|c|c|c|c|c|}
\hline & \multicolumn{2}{|c|}{$\begin{array}{c}\text { Serous } \\
\text { papillary }\end{array}$} & \multicolumn{2}{|c|}{ Clear cell } \\
\hline & No & (\%) & No & (\%) \\
\hline \multicolumn{5}{|l|}{ Age (years) } \\
\hline$<40$ & - & - & 1 & $(9.1)$ \\
\hline $46-74$ & 23 & $(100)$ & 10 & $(90.9)$ \\
\hline \multicolumn{5}{|l|}{ Parity } \\
\hline Nulliparity & 1 & $(4.3)$ & 1 & $(9.1)$ \\
\hline Multiparity & 22 & (95.7) & 10 & $(90.9)$ \\
\hline \multicolumn{5}{|l|}{ Complaint } \\
\hline $\begin{array}{l}\text { Postmenopausal } \\
\text { bleeding }\end{array}$ & 22 & (95.7) & 8 & (72.7) \\
\hline Other & 1 & $(4.3)$ & 3 & $(27.3)$ \\
\hline Diabetes mellitus & 6 & (26.1) & 2 & $(18.2)$ \\
\hline Hypertension & 5 & $(21.7)$ & - & - \\
\hline $\mathrm{DM}+\mathrm{HT}$ & 4 & $(17.3)$ & 1 & (9.1) \\
\hline Breast cancer & 2 & $(8.3)$ & - & - \\
\hline \multicolumn{5}{|l|}{ FIGO stage } \\
\hline IB & 4 & (17.4) & 8 & $(72.7)$ \\
\hline IC & 8 & (34.8) & - & - \\
\hline$\| \mathrm{A}$ & 1 & $(4.3)$ & - & - \\
\hline IIB & 3 & (13.0) & - & - \\
\hline IIIA & 2 & (8.7) & 1 & $(9.1)$ \\
\hline IIIC & 5 & $(21.7)$ & 2 & $(18.2)$ \\
\hline \multicolumn{5}{|l|}{ Myometrial invasion } \\
\hline Yok & - & - & - & - \\
\hline$<1 / 2$ & 7 & (30.4) & 9 & $(81.8)$ \\
\hline$>1 / 2$ & 16 & (69.6) & 2 & $(18.2)$ \\
\hline \multicolumn{5}{|c|}{ Lymphovascular invasion } \\
\hline$(+)$ & 8 & (34.8) & 2 & (18.2) \\
\hline$(-)$ & 15 & $(65.2)$ & 9 & $(81.8)$ \\
\hline \multicolumn{5}{|l|}{ Cervical involvement } \\
\hline$(+)$ & 7 & (30.4) & 3 & $(27.3)$ \\
\hline$(-)$ & 16 & (69.6) & 8 & (72.7) \\
\hline
\end{tabular}




\begin{tabular}{|c|c|c|c|c|}
\hline & \multicolumn{2}{|c|}{$\begin{array}{l}\text { Serous } \\
\text { papillary }\end{array}$} & \multicolumn{2}{|c|}{ Clear cell } \\
\hline & No & (\%) & No & (\%) \\
\hline \multicolumn{5}{|l|}{ Adnexal metastasis } \\
\hline$(+)$ & 4 & $(17.4)$ & - & - \\
\hline$(-)$ & 19 & (82.6) & 11 & $(100)$ \\
\hline \multicolumn{5}{|l|}{ Peritoneal washings } \\
\hline Benign & 23 & $(100)$ & 9 & $(81.8)$ \\
\hline Malign & - & - & 2 & $(18.2)$ \\
\hline \multicolumn{5}{|c|}{ Lymph node involvement } \\
\hline No & 10 & $(43.5)$ & 3 & $(27.3)$ \\
\hline Pelvic & 3 & (13) & 3 & $(27.3)$ \\
\hline Pelvic + paraaortic & 10 & $(43.5)$ & 5 & (45.5) \\
\hline \multicolumn{5}{|l|}{ Grade } \\
\hline Uncertain & 8 & (34.8) & 4 & (36.4) \\
\hline 1 & 5 & (21.7) & - & - \\
\hline 2 & 4 & (17.4) & 3 & $(27.3)$ \\
\hline 3 & 6 & (26.1) & 4 & (36.4) \\
\hline \multicolumn{5}{|l|}{ Adjuvant therapy } \\
\hline No & 1 & $(4.34)$ & 2 & (18.1) \\
\hline ERT & 4 & $(17.3)$ & 1 & $(9.9)$ \\
\hline ERT + IRT & 18 & (78.2) & 8 & (72.7) \\
\hline Radiochemotherapy & 5 & (21.7) & 2 & (18.1) \\
\hline \multicolumn{5}{|l|}{ Survival (months) } \\
\hline OS & \multicolumn{2}{|c|}{$72.7 \pm 52.8$} & \multicolumn{2}{|c|}{$81.7 \pm 40.4$} \\
\hline DFS & \multicolumn{2}{|c|}{$61.6 \pm 56.4$} & \multicolumn{2}{|c|}{$72.8 \pm 48.5$} \\
\hline
\end{tabular}

Sixteen patients had FIGO stage 1-2 and seven patients had FIGO stage 3 in the UPSC group. There were no patients with FIGO stage 2 in the CC group, but 8 patients had FIGO stage 1 and 3 patients had stage 3 disease in the clear cell group. The most common metastatic locations were lung metastases in both groups. The UPSC group had 2 (8.6\%) patients with lung metastases, 1 (4.34\%) with para-aortic metastasis, and 1 (4.34\%) with liver and retroperitoneal metastasis. The CC group had 1 patient (9.09\%) with stump metastases, and 1 patient (9.09\%) with lung metastasis. The surgical stage and morphological characteristics of the patients are shown in Table 1.

Primary total abdominal hysterectomy and bilateral salpingo-oophorectomy, pelvic and para-aortic lymph node excision, and infracolic omentectomy were performed in all cases. All patients received external pelvic radiotherapy except for 1 patient in the serous papillary group and 2 patients in the clear cell group. Recurrence developed in 7 patients, so they received cisplatin $\left(50 \mathrm{mg} / \mathrm{m}^{2}\right)$ and doxorubicin $\left(50 \mathrm{mg} / \mathrm{m}^{2}\right)$ intravenously every 3 weeks. Mean survival rate was $72.7 \pm 52.8$ months and $81.7 \pm 40.4$ months for UPSC and CC groups.

\section{DISCUSSION}

UPSC and CC are highly aggressive tumors, which are characterized independently of endometrial hyperplasia and hyperestrogenemia [5]. These two species are very likely to have extrauterine spread [6]. Even though the primary lesion is very small, early myometrial, lymphovascular, and abdominal lymph node invasion can be seen [7]. Average age of type 2 cancer-affected patients is very high. Bancher-Todesa et al., reported that mean age of patients with UPSC was 72.4 years [8], while Rosenberg et al., reported it to be 69 years [9]. Regardless, mean age of patients in our series was incompatible with the literature. In the UPSC group, 10 patients aged $46-60$ accounted for $43.5 \%$ and 13 patients aged $>61$ accounted for $56.5 \%$ of the cases. In the CC group, there was 1 (9.09\%) patient who was $<40$ years old, 4 (36.4\%) patients aged between 46 and 60, and 6 (54.5\%) patients aged $>61$. In our study, mean age at diagnosis was 59.9 , which is almost 10 years earlier than the mean age reported in the literature.

Postmenopausal bleeding has been reported as the most common symptom in non-endometrioid cancer [10]. Postmenopausal bleeding was identified as the initial symptom in 22 (95.7\%) patients from the UPSC and 11 patients $(72.7 \%)$ with from the CC groups in our study. In a study by Bancher-Todesca et al., postmenopausal bleeding was reported as the initial symptom in 20 out of 23 patients [8]. In our study, obesity, diabetes, and hypertension were not found to be associated with the development of non-endometrioid types of disease. Breast cancer is another factor associated with endometrial cancer, and its prevalence in the general population is $12.2 \%$ [11]. In our study, only 2 patients from the UPSC group (8.3\%) had a history of breast cancer.

Endometrial cancer histological type is one of the most important factors influencing patient survival. In its annual report, FIGO presented five-year survival rates of $83 \%$ for endometrioid histology, $62 \%$ for clear cell histology, and $53 \%$ for serous cell tumors. It is described that even the five-year survival is $90 \%$ for stage I endometrioid histology, $85 \%$ for stage I clear cell histology, and $80 \%$ for stage I serous cell tumors $[12,13]$. Data in our study are consistent with the general literature, but CC groups were found to have a worse prognosis. Endometrioid histology is observed in more early stages but type 2 tumors (serous papillary and clear cell) are often detected at an advanced stage [12]. Mendivil et al., reported that $84 \%$ of patients diagnosed with stage 1-2 for endometrioid histology and this rate is $62 \%$ for serous papillary tumors [14]. In our 
study, 16 patients (69.5\%) were diagnosed at stage 1-2 in the UPSC group, while 8 patients (72.7\%) at stage 1 in the CC group. In contrast to the literature, type 2 tumors were observed at an early stage.

Myometrial invasion is one of the most important independent prognostic factors for endometrial cancer. In several studies, it was reported that superficial invasion was detected in 40-65\%, deep invasion in $10-42 \%$, and no invasion in $10-24 \%$ of the cases $[15,16]$. In our study, all patients presented myometrial invasion. Although superficial myometrial invasion was detected in $30.4 \%$ of the subjects, deep myometrial invasion was detected in $69.6 \%$ of the papillary histological cases. Superficial myometrial invasion was identified as $81.8 \%$, and deep myometrial invasion as $18.2 \%$ in CC. When myometrial invasion was assessed independently of disease stage, it was found to have caused metastasis and reduced disease-free survival in type 2 tumors. In one study, the tumor recurrence rate was only $13 \%$ if the tumors were localized in the fundus, but $44 \%$ if the tumors were localized in the lower uterine segment or the cervix [17]. Cervical involvement was detected in $30.4 \%$ of UPSC patients and $85.7 \%$ of them had stromal invasion. The cervical involvement rate was $27.3 \%$ in the CC group and $66.6 \%$ of them had stromal invasion. The analysis of disease-free survival and overall survival revealed that cervical invasion decreased the survival in both groups.

Lymphovascular invasion is a strong predictor of recurrence. It is independent of the histological grade or myometrial invasion $[18,19]$. The presence of vascular invasion causes lymph node metastasis [20]. In our research, lymph node metastases were detected in $56.5 \%$ of patients with lymphovascular invasion in the UPSC group and $72.8 \%$ of patients with lymphovascular invasion in the CC group. These findings are also supported by the study about lymph node metastases by Cheewakriangkra et al. [21]. Pelvic lymph node involvement was detected in $13 \%$ of 23 patients, pelvic-para-aortic lymph node involvement in $43.5 \%$ of the UPSC group. Eleven patients had $27.3 \%$ pelvic and $45.5 \%$ pelvic-para-aortic lymph node involvement in the CC group. While all patients were evaluated without histology, pelvic metastases proportion was $17.64 \%$, pelvic-para-aortic metastases proportion was $44.11 \%$. In its annual report published in 2006, FIGO reported that the five-year survival rate of patients with stage $3 c$ disease was $57.3 \%$ [12]. In our study, the average overall survival was 43 months in patients who had stage $3 c$ disease in the UPSC and 96 months in the CC groups.

Type II endometrial carcinoma is very susceptible to extrauterine spread. Even if the primary lesion is localized in a very small space, lymph node involvement and distant metastases can be observed [7]. Thus, all type Il endometrial carcinomas should be staged surgically. All patients in our study were subjected to surgical staging. Due to the fact that non-endometrioid tumors have a worse prognosis, all patients, including those in the early stages, should receive adjuvant treatment [22]. However, the efficacy of postoperative adjuvant chemotherapy in these patients remains controversial. Some authors have reported that cisplatin-based chemotherapy provides a cure for non-endometrioid tumors but other authors have reported a relative resistance to chemotherapy [7, 23, 24]. Five (21.7\%) patients with UPSC and 2 (18.1\%) with CC received cisplatin and doxorubicin treatment as adjuvant chemotherapy, but complete response was not observed in either group. Although radiotherapy is a significant adjuvant treatment for endometrioid tumors, the use of adjuvant radiotherapy in non-endometrioid tumors is slightly different $[25,26]$. Bancher-Todesca et al., reported that mean 5-year survival is $30 \%$ in patients with UPSC who were only treated with radiotherapy [8]. On the other hand, Mallipeddi et al., informed about a $50 \%$ survival in 10 patients who were treated by total abdominal radiotherapy [27], but Frank et al., reported that that treatment has no benefit [28]. In our study, the adjuvant radiotherapy was applied to 3 of the 4 patients with stage $1 \mathrm{~b}$ and grade 2 diseases and distant metastases did not occur. Three patients with stage $1 \mathrm{~b}$ grade 3 diseases received adjuvant radiotherapy and brachytherapy and one lung metastasis was observed within a month after the treatment.

\section{CONCLUSIONS}

The stage of disease was seen as the primary factor influencing disease-free and overall survival. In addition, non-endometrioid histology, increased grade, deep myometrial invasion, cervical stoma invasion, presence of malignant cells in cytology, adnexal involvement, lymphovascular invasion, pelvic and/or para-aortic lymph node involvement, and tumor size were found to be effective in survival. We did not achieve statistically significant results to determine whether age, parity, and age at menarche and menopause constitute risk factors for endometrial cancer. Breast cancer was noted more frequently in the UPSC group, which was consistent with the literature. Cisplatin-based chemotherapy was partially effective in patients with postoperative recurrence, but postoperative adjuvant radiotherapy was found to be more effective in preventing relapse. As a result, the history and findings of the patients were analyzed and then compared with the literature. The evaluated parameters were consistent with the literature. We conclude that there is a need for more extensive and multicenter studies to achieve valid results. 


\section{REFERENCES}

1. Bokhman JV.Two pathogenetic types of endometrial carcinoma. Gynecol Oncol. 1983, 15 (1), 10-17.

2. Baltzer J, Lohe KJ, Kuerzl R, [et al.]. Prognostic criteria in patients with endometrial cancer. Arch Gynecol. 1983, 234 (2), 121-129.

3. Kedzia H, Spaczyński M. Uterine papillary serous carcinoma. Ginekol Pol. 1994, 65 (10), 587-592.

4. Olawaiye $A B$, Boruta DM. Management of women with clear cell endometrial cancer: a Society of Gynecologic Oncology (SGO) review. Gynecol Oncol. 2009, 113 (2), 277-283.

5. Moll UM, Chales E, Augeste M, [et al.]. Uterine papillary serous carcinoma evolves via a p53-driven pathway. Hum Pathol. 1996, 1295-1300.

6. Kurman RJ, Scully RE. Clear cell carcinoma of the endometrium: an analysis of 21 cases. Cancer. 1976, 37 (2), 872-882.

7. Goff BA, Kato D, Schmidt RA, [et al.]. Uterine papillary serous carcinoma: patterns of metastatic spread. Gynecol Oncol. 1994, 54, 264-268.

8. Bancher-Todesca D, Neunteufel W, Williams KE, [et al.]. Influence of postoperative treatment on survival in patients with uterine papillary serous carcinoma. Gynecol Oncol. 1998, 71, 344-347.

9. Rosenberg P, Boeryd B, Simonsen E. A new agressive treatment approach to high-grade endometrial cancer of possible benefit to patients with stage I uterine papillary cancer. Gynecol Oncol. 1993, 48, 32-37.

10. Zhang $\mathrm{C}, \mathrm{Hu} \mathrm{W}$, Jia $\mathrm{N}$, [et al.]. Uterine carcinosarcoma and high-risk endometrial carcinomas: a clinicopathological comparison. Int J Gynecol Cancer. 2015, 25 (4), 629-636.

11. Hankey BF, Brinton LA, Kessler LF. Cancer Statistics Review 1973-1990. NIH Publication No 93-2789, 1993.

12. Dunton CJ, Balsara G, McFarland M, [et al.]. Uterine papillary serous carcinoma: a review. Obstet Gynecol Surv. 1991, 46 (2), 97-102.

13. Bidziński $M$, Dańska-Bidzińska A, Derlatka $P$, [et al.]. Recurrence risk analysis in patients treated for I clinical stage of endometrial cancer. Ginekol Pol. 2007, 78 (6), 471-475.

14. Mendivil A, Schuler KM, Gehrig PA. Non-endometrioid adenocarcinoma of the uterine corpus: a review of selected histological subtypes. Cancer Control. 2009, 16 (1), 46-52.
15. Disaia PJ, Creasman WT. Adenocarcinoma of the uterus. Clinical Gynecologic Oncology. $3^{\text {rd }}$ edition. Chapter 19. 1989, 161-197.

16. Sagae S, Susumu N, Viswanathan A, [et al.]. Gynecologic Cancer InterGroup (GCIG) Consensus Review for Uterine Serous Carcinoma. Int J Gynecol Cancer. 2014, 24 (9), 83-89.

17. Disaia PJ, CreasmanWT, Boronow RC, [etal.]. Riskfactors and recurrent patterns in stage 1 endometrial cancer. Am J Obstet Gynecol. 1985, 151, 1009-1015.

18. Morrow $\mathrm{CP}$, Bundy $\mathrm{BN}$, Kurman RJ, [et al.]. Relationship between surgical-pathological risk factors and outcome in clinical stage 1 and 2 carcinoma of the endometrium: a Gynecologic Oncology Group study. Gynecol Oncol. 1991, 40, 55-65.

19. Mariani A, Webb MJ, Keeney GL, [et al.]. Assessment of prognostic factors in stage IIIA endometrial cancer. Gynecol Oncol. 2002, 86, 38-44.

20. Gal D, Recio FO, Zamurovic D, [et al.]. Lymphovascular space involvement: a prognostic indicator inendometrial adenocarcinoma. Gynecol Oncol. 1991, 42, 142-145.

21. Cheewakriangkrai C, Panggid K, Siriaungkul S, [et al.]. Lymphovascular space invasion as a prognostic determinant in uterine cancer. Asian Pac J Cancer Prev. 2007, 8 (3), 363-366.

22. Mahdi H, Rizzo A, Rose PG, [et al.]. Outcome of recurrent uterine papillary serous carcinoma treated with platinum-based chemotherapy. Int J Gynecol Cancer. 2015, 25 (3), 467-473.

23. Gitsch G, Friedlander MJ, Wain GW, Hacker NF. Uterine papillary serous carcinoma: a clinical study. Cancer. 1995, 75, 2239-2243.

24. Smith JP, Rutledge F. Advances in chemotherapy for gynecologic cancer. Obstet Gynecol Surv. 1975, 36 (2), 669-674.

25. Sutton GP, Brill L, Michael $H$, [et al.]. Malignant papillary lesions of the endometrium. Gynecol Oncol. 1987, 27, 294-304.

26. Gallion $\mathrm{HH}$, Van Nageli JR, Powell DF, [et al.]. Stage I serous papillary carcinoma of the endometrium. Cancer. 1989, 63, 2224-2228.

27. Mallipeddi P, Knapp DS, Teng NN. Long-term survival with adjuvant whole abdominopelvic irradiation for uterine papillary serous carcinoma. Cancer. 1993, 71, 3076-3081.

28. Frank AH,Tseng PC, Haffty BG, [et al.]. Adjuvant whole-abdominal radiation therapy in uterine papillary serous carcinoma. Cancer. 1991,68, 1516-1519. 